Supporting information for

\title{
Mechanism and Regioselectivity of Rh(III)-Catalyzed Intermolecular Annulation of Aryl-Substituted Diazenecarboxylates and Alkenes: DFT Insights
}

\author{
Manjaly J. Ajitha and Kuo-Wei Huang* \\ KAUST Catalysis Center and Division of Physical Science and Engineering, \\ King Abdullah University of Science and Technology, Thuwal 23955-6900, Saudi Arabia. \\ *E-mail: hkw@kaust.edu.sa
}

Table S1. Computed energy parameters of important TSs and intermediates.......................S2

Figure S1. Energy profile for the determination of regioselectivity. ................................ 3

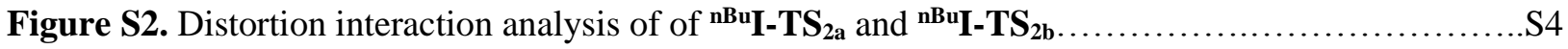


Table S1. Computed energy parameters of important TSs and intermediates.

\begin{tabular}{|c|c|c|c|}
\hline \multirow[t]{2}{*}{ system } & \multicolumn{2}{|c|}{ M062X/gen1 } & \multirow{2}{*}{$\begin{array}{c}\text { M062X/gen } 2 \\
\text { SCF Energy (Hartree }\end{array}$} \\
\hline & SCF Energy (Hartree) & $\mathrm{G}_{\text {corr }}($ Hartree $)$ & \\
\hline I-1 & -1414.78400 & 0.45534 & -1415.24054 \\
\hline $\mathbf{I}-\mathbf{T S}_{\mathbf{1}}$ & -1414.74537 & 0.45213 & -1415.20640 \\
\hline I-TS 1 pdt & -1414.76669 & 0.45862 & -1415.23477 \\
\hline $\mathrm{I}-2$ & -1185.75077 & 0.39880 & -1186.13036 \\
\hline I-TS -rea $_{2}$ & -1264.33339 & 0.45172 & -1264.73352 \\
\hline $\mathbf{I}-\mathbf{T S}_{2}$ & -1264.30510 & 0.45210 & -1264.70535 \\
\hline $\mathbf{I}-\mathbf{3}$ & -1264.33771 & 0.45141 & -1264.73653 \\
\hline II-1 & -1414.78575 & 0.45703 & -1415.24073 \\
\hline II-TS $_{1}$ & -1414.72966 & 0.44870 & -1415.18953 \\
\hline II-TS 1 pdt & -1414.75487 & 0.45306 & -1415.21268 \\
\hline II-2 & -1185.73245 & 0.39643 & -1186.11154 \\
\hline II-TS 2 rea & -1264.30727 & 0.44917 & -1264.70782 \\
\hline II-TS 2 & -1264.28411 & 0.44961 & -1264.68373 \\
\hline II-3 & -1264.34967 & 0.45488 & -1264.74567 \\
\hline $\mathbf{I}-\mathbf{T S}_{\mathbf{3 a}}$ & -1264.28772 & 0.45250 & -1264.68761 \\
\hline I-TS ${ }_{3 a}^{\prime}$ rea & -1493.35381 & 0.51433 & -1493.83699 \\
\hline $\mathbf{I}^{-T S_{3 a}}{ }^{\prime}$ & -1493.30703 & 0.51212 & -1493.78738 \\
\hline $\mathbf{T S}_{\mathrm{cs}}$ & -1264.31583 & 0.45351 & -1264.71233 \\
\hline I-TS* & -1264.30191 & 0.45049 & -1264.72356 \\
\hline $\mathbf{I}-3^{*}$ & -1264.32345 & 0.44945 & -1264.72612 \\
\hline I-TS ${ }^{* *}$ & -1264.26876 & 0.45010 & -1264.67905 \\
\hline $\mathbf{I}-\mathbf{T S}_{\mathbf{3 b}}$ & -1264.32269 & 0.45426 & -1264.72740 \\
\hline I-4 & -1264.36321 & 0.45848 & -1264.78251 \\
\hline I-TS 4 rea & -1493.39111 & 0.51459 & -1493.87991 \\
\hline $\mathrm{I}_{-} \mathbf{T S}_{4}$ & -1493.38931 & 0.51285 & -1493.87776 \\
\hline I-5 & -1493.39899 & 0.51768 & -1493.89174 \\
\hline I-TS ${ }_{3 b}$ 'rea & -1493.35722 & 0.51128 & -1493.83664 \\
\hline $\mathbf{I}-\mathbf{T S}_{\mathbf{3 b}}{ }^{\prime}$ & -1493.29760 & 0.50943 & -1493.77879 \\
\hline${ }^{\mathrm{CN}} \mathbf{I}-\mathbf{T S}_{2 \mathrm{a}}$ rea & -1356.53933 & 0.44980 & -1356.97567 \\
\hline${ }^{\mathrm{CN}^{-}} \mathbf{I}-\mathbf{T S}_{2 \mathrm{a}}$ & -1356.51748 & 0.44910 & -1356.95525 \\
\hline${ }^{\mathbf{C N}} \mathbf{I}-\mathbf{3}_{\mathrm{a}}$ & -1356.55608 & 0.45129 & -1356.99070 \\
\hline${ }^{\mathrm{CN}} \mathbf{I}-\mathbf{T S}_{2 \mathrm{~b}}$ rea & -1356.54253 & 0.44962 & -1356.97667 \\
\hline${ }^{\mathrm{CN}} \mathbf{I}-\mathbf{T S}_{2 \mathrm{~b}}$ & -1356.50722 & 0.44969 & -1356.94277 \\
\hline${ }^{\mathrm{CN}} \mathbf{I}-\mathbf{3}_{\mathrm{b}}$ & -1356.53657 & 0.44911 & -1356.97709 \\
\hline${ }^{\text {nBu }}{ }_{I-T S}{ }_{2 a}$ rea & -1421.51038 & 0.56149 & -1421.95242 \\
\hline${ }^{\mathrm{nBu}} \mathbf{I}-\mathbf{T S}_{2 \mathrm{a}}$ & -1421.48296 & 0.56018 & -1421.92598 \\
\hline${ }^{n B u} \mathbf{I}-3_{a}$ & -1421.51213 & 0.56176 & -1421.95331 \\
\hline${ }^{\text {nBu}} \mathbf{I}-T_{S_{2 b}}$ rea & -1421.51030 & 0.56014 & -1421.95356 \\
\hline${ }^{\mathrm{nBu}} \mathbf{I}-\mathrm{TS}_{2 \mathrm{~b}}$ & -1421.48157 & 0.56238 & -1421.92773 \\
\hline${ }^{n B u} \mathbf{I}-3_{b}$ & -1421.50703 & 0.56180 & -1421.94761 \\
\hline
\end{tabular}


Figure S1. Energy profile for the determination of regioselectivity. The net charge of each species is +1 .
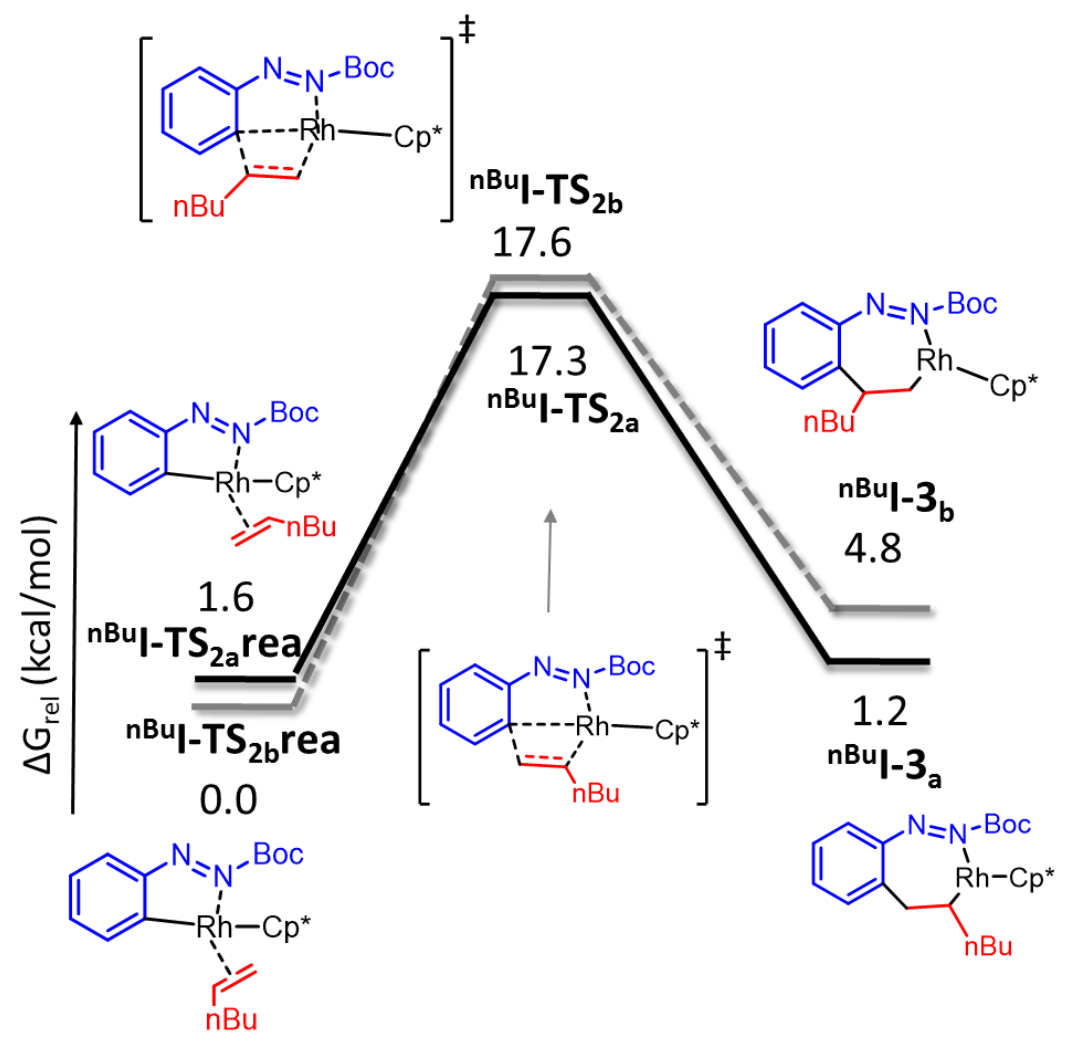
Figure S2. Distortion interaction analysis of of ${ }^{\mathbf{n B u}} \mathbf{I}-\mathbf{T S}_{\mathbf{2 a}}$ and ${ }^{\mathbf{n B u}} \mathbf{I}-\mathbf{T S}_{\mathbf{2 b}}$.

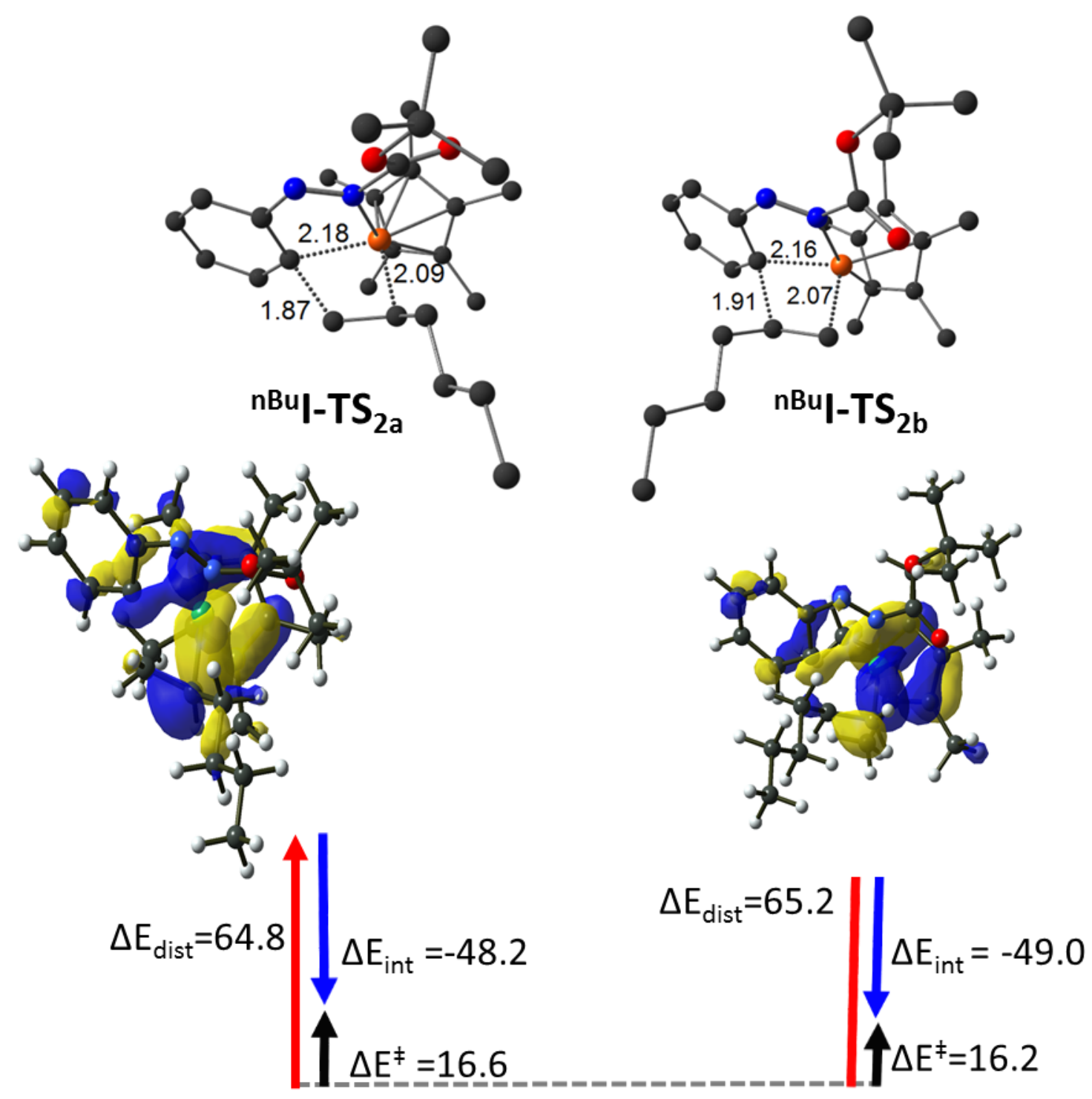

